\title{
Non-Invasive Hemodynamic Monitoring for Hemodynamic Management in Perioperative Medicine
}

\author{
Julia Y. Nicklas and Bernd Sauge/* \\ Department of Anesthesiology, Center of Anesthesiology and Intensive Care Medicine, University Medical Center Hamburg- \\ Eppendorf, Hamburg, Germany
}

Keywords: cardiovascular monitoring, blood pressure, advanced hemodynamic variables, continuous cardiac output, pulse contour analysis

\section{BACKGROUND}

OPEN ACCESS

Edited by:

Mikhail Kirov,

Northern State Medical University,

Russia

Reviewed by:

Alexey Smetkin,

Northern State Medical University,

Russia

Vladimir Lomivorotov,

Novosibirsk Scientific Research Institute of Pathology, Russia

${ }^{*}$ Correspondence:

Bernd Saugel

bcs.muc@gmx.de

Specialty section:

This article was submitted

to Intensive Care Medicine

and Anesthesiology,

a section of the journal

Frontiers in Medicine

Received: 09 October 2017 Accepted: 08 November 2017 Published: 23 November 2017

Citation:

Nicklas JY and Saugel B (2017) Non-Invasive Hemodynamic Monitoring for Hemodynamic Management in Perioperative

Medicine.

Front. Med. 4:209

doi: 10.3389/fmed.2017.00209
In perioperative medicine, hemodynamic management aims at an optimization of perfusion pressure and oxygen delivery in order to maintain or restore adequate cellular metabolism (1). To optimize cardiopulmonary function, hemodynamic management triggers the administration of fluids and vasoactive agents according to predefined target values of hemodynamic variables. This is often referred to as "goal-directed therapy" (GDT). Although the general and vague term GDT comprises various (in part very different) hemodynamic treatment strategies (2), GDT has been shown to improve patient outcome, especially in high-risk patients undergoing major surgery (3-11). Besides basic hemodynamic variables (blood pressure and heart rate), GDT treatment algorithms usually include advanced hemodynamic variables such as pressure- or volume-based cardiac preload variables (central venous pressure, pulmonary capillary wedge pressure, global end-diastolic volume), dynamic cardiac preload variables (pulse pressure variation, stroke volume variation), and blood flow variables (stroke volume, cardiac output). A variety of invasive, less-invasive, and non-invasive hemodynamic monitoring technologies are nowadays available to assess hemodynamic variables in the operating room or the intensive care unit. In this opinion paper, we will discuss how innovative non-invasive hemodynamic monitoring might be used for hemodynamic management in perioperative medicine.

\section{HEMODYNAMIC MONITORING TECHNOLOGIES USED FOR GDT}

Until recently, the measurement of advanced hemodynamic variables used in GDT protocols required invasive hemodynamic monitoring such as invasive pulse contour analysis (arterial catheter), transpulmonary thermodilution (dedicated arterial catheter and central venous catheter), or pulmonary artery thermodilution (pulmonary artery catheter). However, during the last decades, the use of the pulmonary artery catheter in perioperative medicine and critical care is declining $(12,13)$ and the routine use of the pulmonary artery catheter is not recommended for surgical patients undergoing non-cardiac surgery (14). Advanced hemodynamic monitoring using the transpulmonary thermodilution technique, often called a less-invasive alternative to the pulmonary artery catheter, is also used only in a minority of patients in the perioperative period (15). Especially in the UK, the esophageal doppler is used to assess blood flow for perioperative GDT (3). Many recent studies on perioperative GDT used un-calibrated invasive pulse contour analysis (arterial catheter) to assess blood pressure, dynamic cardiac preload parameters, or cardiac output $(3,4,16-18)$. 
In the recent years, different completely non-invasive hemodynamic monitoring technologies were proposed (19). Measurement principles of these innovative hemodynamic monitoring technologies are, among others, bioimpedance and bioreactance, pulse wave transit time, partial carbon dioxide rebreathing, and non-invasive pulse contour analysis (19-27). It is beyond the scope of this article to discuss in detail the underlying measurement principles. In general, the main advantage of these new technologies is that they allow the estimation of cardiac output and other advanced hemodynamic variables without the need for arterial or central venous cannulation. In addition, using these technologies in clinical practice is relatively easy and does not require extensive training. On the other hand, all of the available technologies still have technical limitations with regard to their clinical applicability (19). Furthermore, the numerous validation studies comparing these innovative measurement technologies with established reference methods revealed contradicting results $(19,28-31)$.

In the following, we want to describe how these innovative technologies can be used for hemodynamic management in perioperative medicine.

\section{NON-INVASIVE HEMODYNAMIC MONITORING FOR PERIOPERATIVE HEMODYNAMIC MANAGEMENT- AVAILABLE DATA}

There are still only a few studies available that investigated the feasibility and usefulness of perioperative GDT based on completely non-invasive hemodynamic monitoring technologies.

In a prospective randomized controlled trial, Benes et al. (32) evaluated the impact of continuous non-invasive blood pressure monitoring using the volume clamp method (finger cuff) on blood pressure stability in patients undergoing thyroid gland surgery in an upright position ("beach chair position"). Patients were randomized to the study group (continuous blood pressure monitoring) or to the control group (intermittent blood pressure monitoring with oscillometric upper arm cuff). Continuous non-invasive blood pressure monitoring significantly decreased the time spent in intraoperative hypotension defined as blood pressure $-20 \%$ below the individual patient's target blood pressure ( 14 vs. $34 \%$; $p=0.003$ ). However, the study was too small to adequately evaluate whether this reduction of time spent in hypotension translates into an improvement in postoperative patient outcome.

Fellahi and colleagues (33) evaluated the impact of intraoperative GDT based on stroke volume variation and cardiac index assessed with an endotracheal bioimpedance cardiac output monitor on postoperative outcome after coronary artery bypass surgery in a prospective, controlled, parallel-arm trial. In patients in the study group, the proportion of patients receiving fluid loading and dobutamine was higher compared with the control group. Although the primary endpoint (time to hospital discharge) was not different between the groups, the time to extubation was statistically significantly shorter in the GDT intervention group.

In a similar setting, Leclercq et al. (34) evaluated the feasibility and clinical utility of an endotracheal bioimpedance cardiac output monitoring to optimize intraoperative hemodynamics and improve short-term outcome in patients undergoing off-pump coronary artery bypass grafting surgery. The authors compared 20 patients in whom hemodynamics were monitored with the bioimpedance technology with a historic control of 42 patients. The primary endpoint, the rate of postoperative intensive care unit admission, occurred significantly less often in the bioimpedance group than in the control group ( 55 vs. $90 \% ; p=0.008$ ). In addition, the time to extubation, the length of stay in the intensive care unit, and the lactate level $6 \mathrm{~h}$ after surgery were significantly lower in the bioimpedance group. The authors thus concluded that the systematic use of endotracheal bioimpedance cardiac output monitoring is associated with a reduction in the rate of intensive care unit admission and an improvement in immediate postoperative outcome in patients undergoing off-pump coronary artery bypass grafting surgery.

Broch et al. (35) investigated the feasibility and clinical impact (postoperative complications up to 28 days and length of hospital stay) of GDT based on non-invasive pulse contour analysis (volume clamp method) in patients undergoing elective major abdominal surgery. In their randomized controlled trial, patients in the study group who were treated according to an algorithm based on non-invasively assessed cardiac index and pulse pressure variation were compared with patients in the control group ("standard of care"). The total number of complications was lower in the study group compared with the control group without reaching statistical significance (94 vs. $132 ; p=0.22$ ). There was also no clinically relevant or statistically significant difference in hospital length of stay or mortality. Thus, the authors conclude that this study demonstrates the general feasibility of a non-invasive GDT approach for hemodynamic optimization in major abdominal surgery. However, following this specific GDT protocol did not improve outcome.

The pleth variability index (i.e., the variability in the pulse oximeter plethysmogram) can be used as a non-invasive dynamic cardiac preload parameter. Forget et al. (36) randomized 82 major abdominal surgery patients into two groups to compare intraoperative fluid management guided by the pleth variability index and mean arterial pressure vs. standard fluid management based on mean arterial and central venous pressure. Interestingly, the amount of intraoperatively administered crystalloids and the total volume of fluids infused were significantly lower in the pleth variability index-GDT group. Lactate levels (primary endpoint) were significantly lower in the GDT group compared with the control group during surgery and $48 \mathrm{~h}$ after surgery.

In the multicenter (six tertiary hospitals) randomized clinical POEMAS trial (37), it was evaluated whether perioperative GDT based on non-invasive bioreactance monitoring decreases the incidence of postoperative complications and hospital length of stay in 142 major abdominal surgery patients requiring intensive care unit admission. The GDT protocol included the administration of fluids and vasoactive agents to target values for mean arterial pressure and cardiac index. In the study group, colloid boluses, red blood cell units, and dobutamine was used more often compared with the control group. The study failed to demonstrate a beneficial impact of GDT on patient outcome 


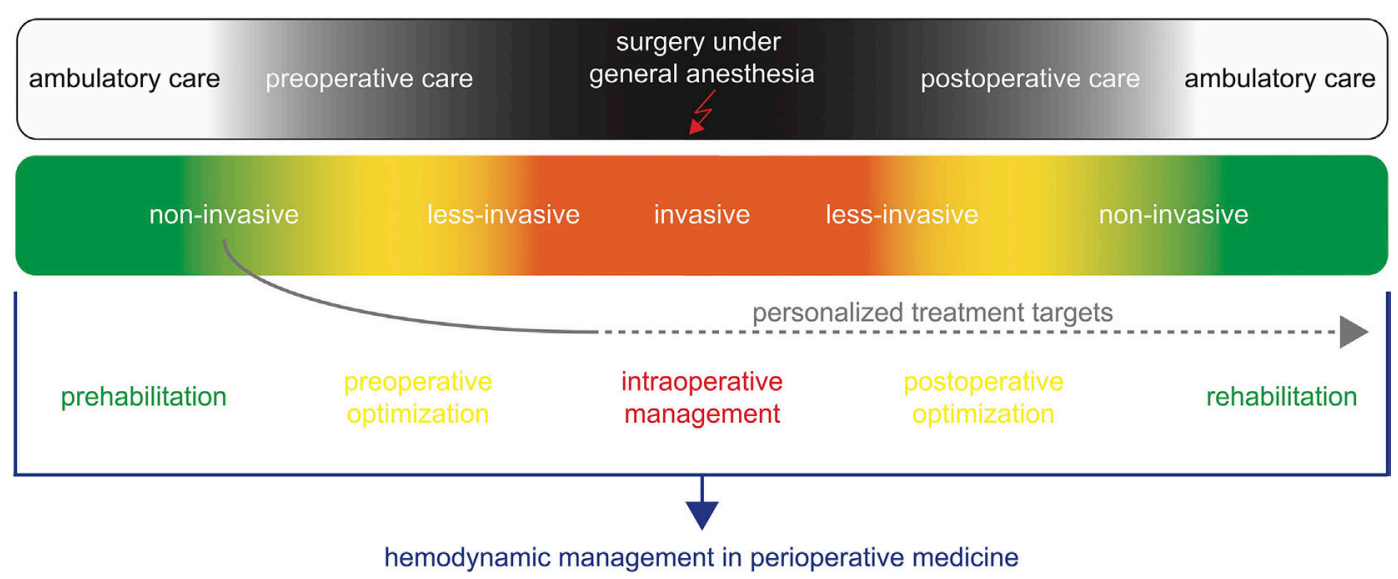

FIGURE 1 | Non-invasive hemodynamic monitoring for hemodynamic management in perioperative medicine. Non-invasive hemodynamic monitoring might be applied for prehabilitation and preoperative optimization during the ambulatory and preoperative care. In addition, it can be used to define personalized targets for the intraoperative hemodynamic management and postoperative optimization.

in terms of overall complications or length of stay between the intervention group and the control group.

We soon will report the results of a monocenter randomized controlled trial in high-risk patients undergoing major abdominal surgery (https://clinicaltrials.gov: NCT02834377) in which we performed "personalized hemodynamic management" (1) by applying a protocol for intraoperative GDT to target the patients' personal normal cardiac index values as measured the day before surgery using the non-invasive volume clamp method.

\section{NON-INVASIVE HEMODYNAMIC MONITORING FOR PERIOPERATIVE HEMODYNAMIC MANAGEMENT - FUTURE PERSPECTIVES}

As discussed above, to date, there are still limited data on the use of non-invasive hemodynamic monitoring technologies for perioperative GDT.

Nevertheless, in the future, these innovative technologies for continuous non-invasive advanced hemodynamic monitoring might offer a variety of opportunities to improve and expand perioperative GDT strategies.

Non-invasive monitoring technologies might enable hemodynamic management strategies to be applied in different new clinical settings (intermediate and low risk surgery) and in patient groups in which advanced hemodynamic monitoring was so far usually not applied (e.g., in patients without arterial catheter or in patients undergoing surgery in regional peripheral or neuraxial anesthesia).

In addition, with non-invasive monitoring technologies, the patients' hemodynamic status can be assessed even before induction of anesthesia and after surgery (Figure 1). Noninvasive hemodynamic monitoring might thus be applied for prehabilitation [i.e., optimizing the patient's hemodynamic status in the weeks before surgery (38)] and preoperative optimization. In addition, values of hemodynamic variables assessed at different time points in the preoperative phase might be used as targets to guide intraoperative hemodynamic management and postoperative optimization (1). The availability of non-invasive technologies for the assessment of advanced hemodynamic variables might thus open a window for perioperative concepts of "personalized hemodynamic management" that aims to optimize cardiovascular dynamics based on the patient's personal hemodynamic profile (1). Because these innovative technologies enable blood pressure, blood flow, and dynamic cardiac preload variables to be estimated in a completely non-invasive manner even in the preoperative evaluation clinic or on the normal ward, they can be used to determine a patient's personal normal values of these hemodynamic variables prior to induction of anesthesia and surgery (1). Thus, non-invasive hemodynamic monitoring technologies might help to assess and define personal target values for perioperative GDT in contrast to conventional GDT often using predefined fixed population-based "normal" values as hemodynamic target values (1).

In the future, further technical and digital innovations [e.g., implantable, wireless, or wearable sensors $(39,40)]$ might further pave the way for GDT based on non-invasive hemodynamic monitoring in perioperative medicine.

\section{CONCLUSION}

Perioperative hemodynamic management based on the assessment of advanced hemodynamic variables aims at an optimization of cardiovascular dynamics to improve postoperative patient outcome. Until recently, hemodynamic management required invasive hemodynamic monitoring (arterial catheter, central venous catheter, pulmonary artery catheter). Recently, different monitoring technologies that enable advanced hemodynamic variables to be estimated non-invasively became available. In theory, these innovative technologies for continuous non-invasive advanced hemodynamic monitoring might offer a variety of opportunities to improve and expand hemodynamic 
management strategies and to personalize hemodynamic management (1) in the perioperative phase. However, there are still only a few studies available investigating perioperative GDT based on these innovative technologies with regard to the clinical feasibility and the impact on patient outcome; thus, further research is needed to evaluate and establish non-invasive hemodynamic monitoring for hemodynamic management in perioperative medicine.

\section{REFERENCES}

1. Saugel B, Vincent JL, Wagner JY. Personalized hemodynamic management. Curr Opin Crit Care (2017) 23:334-41. doi:10.1097/MCC.0000000000000422

2. Roche AM, Miller TE. Goal-directed or goal-misdirected - how should we interpret the literature? Crit Care (2010) 14:129. doi:10.1186/cc8884

3. Sun Y, Chai F, Pan C, Romeiser JL, Gan TJ. Effect of perioperative goal-directed hemodynamic therapy on postoperative recovery following major abdominal surgery-a systematic review and meta-analysis of randomized controlled trials. Crit Care (2017) 21:141. doi:10.1186/s13054-017-1728-8

4. Michard F, Giglio MT, Brienza N. Perioperative goal-directed therapy with uncalibrated pulse contour methods: impact on fluid management and postoperative outcome. Br J Anaesth (2017) 119:22-30. doi:10.1093/bja/aex138

5. Osawa EA, Rhodes A, Landoni G, Galas FR, Fukushima JT, Park CH, et al. Effect of perioperative goal-directed hemodynamic resuscitation therapy on outcomes following cardiac surgery: a randomized clinical trial and systematic review. Crit Care Med(2016) 44:724-33. doi:10.1097/CCM.0000000000001479

6. Benes J, Giglio M, Brienza N, Michard F. The effects of goal directed fluid therapy based on dynamic parameters on post-surgical outcome: a meta-analysis of randomized controlled trials. Crit Care (2014) 18:584. doi:10.1186/s13054-014-0584-z

7. Cecconi M, Corredor C, Arulkumaran N, Abuella G, Ball J, Grounds RM, et al. Clinical review: goal-directed therapy-what is the evidence in surgical patients? The effect on different risk groups. Crit Care (2013) 17:209. doi:10.1186/cc11823

8. Grocott MP, Dushianthan A, Hamilton MA, Mythen MG, Harrison D, Rowan K, et al. Perioperative increase in global blood flow to explicit defined goals and outcomes following surgery. Cochrane Database Syst Rev (2012) 11:CD004082. doi:10.1002/14651858.CD004082.pub5

9. Aya HD, Cecconi M, Hamilton M, Rhodes A. Goal-directed therapy in cardiac surgery: a systematic review and meta-analysis. Br J Anaesth (2013) 110:510-7. doi:10.1093/bja/aet020

10. Hamilton MA, Cecconi M, Rhodes A. A systematic review and meta-analysis on the use of preemptive hemodynamic intervention to improve postoperative outcomes in moderate and high-risk surgical patients. Anesth Analg (2011) 112:1392-402. doi:10.1213/ANE.0b013e3181eeaae5

11. Gurgel ST, do Nascimento P Jr. Maintaining tissue perfusion in high-risk surgical patients: a systematic review of randomized clinical trials. Anesth Analg (2011) 112:1384-91. doi:10.1213/ANE.0b013e3182055384

12. Seifi A, Elliott RJ, Elsehety MA. Usage of Swan-Ganz catheterization during the past 2 decades in United States. J Crit Care (2016) 35:213-4. doi:10.1016/j. jcrc.2016.05.024

13. Wiener RS, Welch HG. Trends in the use of the pulmonary artery catheter in the United States, 1993-2004. JAMA (2007) 298:423-9. doi:10.1001/ jama.298.4.423

14. Kristensen SD, Knuuti J, Saraste A, Anker S, Botker HE, Hert SD, et al. 2014 ESC/ESA guidelines on non-cardiac surgery: cardiovascular assessment and management: the joint task force on non-cardiac surgery: cardiovascular assessment and management of the European Society of Cardiology (ESC) and the European Society of Anaesthesiology (ESA). Eur Heart J (2014) 35:2383-431. doi:10.1093/eurheartj/ehu282

15. Funcke S, Sander M, Goepfert MS, Groesdonk H, Heringlake M, Hirsch J, et al. Practice of hemodynamic monitoring and management in German, Austrian, and Swiss intensive care units: the multicenter cross-sectional ICU-CardioMan Study. Ann Intensive Care (2016) 6:49. doi:10.1186/s13613-016-0148-2

\section{AUTHOR CONTRIBUTIONS}

JYN and BS both contributed substantially to the conception of the work and drafted the manuscript. The authors agree to be accountable for all aspects of the work and ensure that questions related to the accuracy or integrity of any part of the work were appropriately investigated. JYN and BS approved the final version of the manuscript to be published.

16. Salzwedel C, Puig J, Carstens A, Bein B, Molnar Z, Kiss K, et al. Perioperative goal-directed hemodynamic therapy based on radial arterial pulse pressure variation and continuous cardiac index trending reduces postoperative complications after major abdominal surgery: a multi-center, prospective, randomized study. Crit Care (2013) 17:R191. doi:10.1186/cc12885

17. Pearse RM, Harrison DA, MacDonald N, Gillies MA, Blunt M, Ackland G, et al. Effect of a perioperative, cardiac output-guided hemodynamic therapy algorithm on outcomes following major gastrointestinal surgery: a randomized clinical trial and systematic review. JAMA (2014) 311:2181-90. doi:10.1001/ jama.2014.5305

18. Malbouisson LMS, Silva JM Jr, Carmona MJC, Lopes MR, Assuncao MS, Valiatti J, et al. A pragmatic multi-center trial of goal-directed fluid management based on pulse pressure variation monitoring during high-risk surgery. BMC Anesthesiol (2017) 17:70. doi:10.1186/s12871-017-0356-9

19. Saugel B, Cecconi M, Wagner JY, Reuter DA. Noninvasive continuous cardiac output monitoring in perioperative and intensive care medicine. Br J Anaesth (2015) 114:562-75. doi:10.1093/bja/aeu447

20. Saugel B, Dueck R, Wagner JY. Measurement of blood pressure. Best Pract Res Clin Anaesthesiol (2014) 28:309-22. doi:10.1016/j.bpa.2014.08.001

21. Sangkum L, Liu GL, Yu L, Yan H, Kaye AD, Liu H. Minimally invasive or noninvasive cardiac output measurement: an update. J Anesth (2016). doi:10.1007/ s00540-016-2154-9

22. Jakovljevic DG, Trenell MI, MacGowan GA. Bioimpedance and bioreactance methods for monitoring cardiac output. Best Pract Res Clin Anaesthesiol (2014) 28:381-94. doi:10.1016/j.bpa.2014.09.003

23. Fellahi JL, Fischer MO. Electrical bioimpedance cardiography: an old technology with new hopes for the future. J Cardiothorac Vasc Anesth (2014) 28:755-60. doi:10.1053/j.jvca.2013.12.026

24. Chamos C, Vele L, Hamilton M, Cecconi M. Less invasive methods of advanced hemodynamic monitoring: principles, devices, and their role in the perioperative hemodynamic optimization. Perioper Med (Lond) (2013) 2:19. doi:10.1186/2047-0525-2-19

25. Marik PE. Noninvasive cardiac output monitors: a state-of the-art review. J Cardiothorac Vasc Anesth (2013) 27:121-34. doi:10.1053/j.jvca.2012.03.022

26. Thiele RH, Bartels K, Gan TJ. Cardiac output monitoring: a contemporary assessment and review. Crit Care Med (2015) 43:177-85. doi:10.1097/ CCM.0000000000000608

27. Renner J, Grunewald M, Bein B. Monitoring high-risk patients: minimally invasive and non-invasive possibilities. Best Pract Res Clin Anaesthesiol (2016) 30:201-16. doi:10.1016/j.bpa.2016.04.006

28. Saugel B, Wagner JY. Innovative noninvasive hemodynamic monitoring: curb your enthusiasm after initial validation studies and evaluate the technologies' clinical applicability. J Clin Monit Comput (2016) 30:509-10. doi:10.1007/ s10877-016-9852-6

29. Wagner JY, Saugel B. When should we adopt continuous noninvasive hemodynamic monitoring technologies into clinical routine? J Clin Monit Comput (2014) 29:1-3. doi:10.1007/s10877-014-9619-x

30. Saugel B, Reuter DA. Are we ready for the age of non-invasive haemodynamic monitoring? Br J Anaesth (2014) 113:340-3. doi:10.1093/bja/aeu145

31. Joosten A, Desebbe O, Suehiro K, Murphy LS, Essiet M, Alexander B, et al. Accuracy and precision of non-invasive cardiac output monitoring devices in perioperative medicine: a systematic review and meta-analysisdagger. Br J Anaesth (2017) 118:298-310. doi:10.1093/bja/aew461

32. Benes J, Simanova A, Tovarnicka T, Sevcikova S, Kletecka J, Zatloukal J, et al. Continuous non-invasive monitoring improves blood pressure stability in 
upright position: randomized controlled trial. J Clin Monit Comput (2014) 29:11-7. doi:10.1007/s10877-014-9586-2

33. Fellahi JL, Brossier D, Dechanet F, Fischer MO, Saplacan V, Gerard JL, et al. Early goal-directed therapy based on endotracheal bioimpedance cardiography: a prospective, randomized controlled study in coronary surgery. J Clin Monit Comput (2014) 29:351-8. doi:10.1007/s10877-014-9611-5

34. Leclercq T, Lilot M, Schulz T, Meyer A, Farhat F, Fellahi JL. Endotracheal bioimpedance cardiography improves immediate postoperative outcome: a case-control study in off-pump coronary surgery. J Clin Monit Comput (2017). doi:10.1007/s10877-017-9996-z

35. Broch O, Carstens A, Gruenewald M, Nischelsky E, Vellmer L, Bein B, et al. Non-invasive hemodynamic optimization in major abdominal surgery: a feasibility study. Minerva Anestesiol (2016) 82:1158-69.

36. Forget $\mathrm{P}$, Lois F, de Kock M. Goal-directed fluid management based on the pulse oximeter-derived pleth variability index reduces lactate levels and improves fluid management. Anesth Analg (2010) 111:910-4. doi:10.1213/ ANE.0b013e3181eb624f

37. Pestana D, Espinosa E, Eden A, Najera D, Collar L, Aldecoa C, et al. Perioperative goal-directed hemodynamic optimization using noninvasive cardiac output monitoring in major abdominal surgery: a prospective, randomized, multicenter, pragmatic trial: POEMAS study (PeriOperative goal-directed thErapy in Major Abdominal Surgery). Anesth Analg (2014) 119:579-87. doi:10.1213/ANE.0000000000000295

38. Michard F, Gan TJ, Kehlet H. Digital innovations and emerging technologies for enhanced recovery programmes. Br J Anaesth (2017) 119(1):31-9. doi:10.1093/bja/aex140
39. Michard F. A sneak peek into digital innovations and wearable sensors for cardiac monitoring. JClin Monit Comput (2017) 31:253-9. doi:10.1007/ s10877-016-9925-6

40. Michard F. Hemodynamic monitoring in the era of digital health. Ann Intensive Care (2016) 6:15. doi:10.1186/s13613-016-0119-7

Conflict of Interest Statement: JYN received institutional research grants, unrestricted research grants, and refunds of travel expenses from Tensys Medical Inc. (San Diego, CA, USA). JYN received refunds of travel expenses from CNSystems Medizintechnik AG (Graz, Austria). BS collaborates with Pulsion Medical Systems SE (Feldkirchen, Germany) as member of the medical advisory board and received honoraria for giving lectures and refunds of travel expenses from Pulsion Medical Systems SE. BS received institutional research grants, unrestricted research grants, and refunds of travel expenses from Tensys Medical Inc. (San Diego, CA, USA). BS received honoraria for giving lectures and refunds of travel expenses from CNSystems Medizintechnik AG (Graz, Austria). BS received research support from Edwards Lifesciences (Irvine, CA, USA).

The reviewer AS and handling Editor declared their shared affiliation.

Copyright $\odot 2017$ Nicklas and Saugel. This is an open-access article distributed under the terms of the Creative Commons Attribution License (CC BY). The use, distribution or reproduction in other forums is permitted, provided the original author(s) or licensor are credited and that the original publication in this journal is cited, in accordance with accepted academic practice. No use, distribution or reproduction is permitted which does not comply with these terms. 